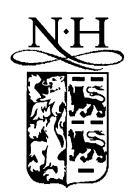

ELSEVIER

\title{
On the states of orientations along a magnetically inhomogeneous nanowire
}

\author{
M. Eisenbach*, M. Dijkstra, B.L. Györffy \\ H.H. Wills Physics Laboratory, University of Bristol, Tyndall Avenue, Bristol, BS8 ITL, UK \\ Received 10 March 1999; received in revised form 5 August 1999
}

\begin{abstract}
We study a simple model for a wire which consists of alternate magnetic and non-magnetic segments. We are interested in the state of relative orientations of the disc-like magnetic segments. In particular, we investigated the ground state and the finite temperature phase diagram of the system, using ground state search, Mean field approximation and Monte Carlo simulations. We find a rich variety of orientational transitions. (C) 2000 Elsevier Science B.V. All rights reserved.
\end{abstract}

Keywords: Nanowires; Dipolar interaction; Layered structures

\section{Introduction}

When a magnet is of mesoscopic size in one or two dimensions, as in the case of ultra-thin films or nanowires, magnetic anisotropy plays a more important role than in bulk samples. Of particular current interest are the orientational transitions in magnetic thin films and multi-layers [1]. In this paper we will attempt to show that quasi-onedimensional structures can exhibit similar and equally rich magnetic behaviour.

In short, we will examine a simple classical spinmodel for layered nanowires, which, due to advances in production techniques, can be manufactured quite readily [2]. We will assume that these wires may be represented as an infinite stack of alternating ferromagnetic and non-magnetic metallic cylinders as shown in Fig. 1, and study the relative magnetic orientation of the discs.

\footnotetext{
* Corresponding author.
}

For an initial exploration of the subject we shall assume that the exchange interaction in the disc is much stronger than between the discs and simplify the model further to that of a chain of classical magnetic moments of fixed magnitude $M$. Namely, we take the $i$ th disc to have a magnetization of $M \boldsymbol{s}_{i}$, where $M$ is identical for all discs and independent of the direction. The direction is given by the classical unit vector $\boldsymbol{s}_{i}$. To simplify the formulae the magnetic moment $M$ will be absorbed into the coefficients $J, K$ and $\omega$ to be defined below. Namely, we investigate the consequences of the following simple classical spin Hamiltonian:

$H=\sum_{i}\left(h_{i}^{\mathrm{ex}}+h_{i}^{\mathrm{A}}+h_{i}^{\mathrm{D}}\right)$,

where

$h_{i}^{\mathrm{ex}}=-J \boldsymbol{s}_{i-1} \cdot \boldsymbol{s}_{i}$

is the exchange interaction between two neighbouring ferromagnetic discs mediated across the intermediate non-magnetic layer. The physical origin of 


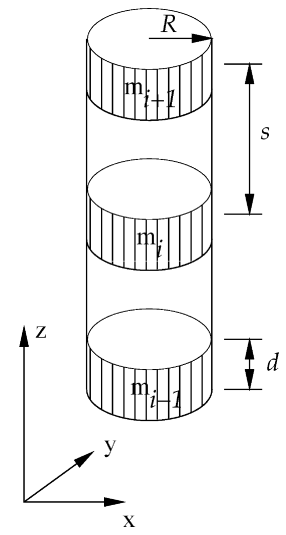

Fig. 1. Geometry of a layered nanowire. The radius of the wire is denoted by $R$, the thickness of the ferromagnetic discs by $d$ and the separation by $s$.

the exchange interaction in such discs is a much studied subject and is well understood in the context of metallic multilayer systems [3]. But, as yet, we are not striving for a realistic description and therefore regard $J$ as an adjustable parameter, independent of the disc separation $s$.

$h_{i}^{\mathrm{A}}=-K\left[\left(s_{i}^{x}\right)^{2}+\left(s_{i}^{y}\right)^{2}\right]$,

represents the shape anisotropy of the ferromagnetic discs, and finally $h^{\mathrm{D}}$ is the dipolar interaction between the ferromagnetic discs of the wire, given by

$h_{i}^{\mathrm{D}}=\omega \sum_{i \neq j}\left(\frac{\boldsymbol{s}_{i} \cdot \boldsymbol{s}_{j}}{|i-j|^{3}}-3 \frac{s_{i}^{z} s_{j}^{z}}{|i-j|^{3}}\right)$.

Note that the shape anisotropy causes the magnetization in the discs, depending on the ratio of the radius $R$ and the thickness $d$, to be aligned, preferentially, parallel or perpendicular to their axis. Our principal interest will focus on the magnetic reorientation of such discs due to their mutual interaction via $h_{i}^{\text {ex }}$ and $h_{i}^{\text {D }}$.

\section{Ground state}

Let us begin by investigating states for which all spins point in the same direction in the $x-z$ plane. Namely, we take

$\boldsymbol{s}_{i} \in\left\{ \pm \boldsymbol{e}_{x}, \pm \boldsymbol{e}_{z}\right\}$ for all $i$. With this restriction the energies of different configurations, can be readily calculated and their relative stability can be determined analytically.

If the anisotropy favours the magnetic moments of the individual discs to be aligned parallel to the chain axis, the ground state will always be the state where the moments are aligned 'head to tail' along the $z$-axis. The energy per site, then, is given by

$E^{\mathrm{Ax}}=-J-K-4 \omega \zeta(3)$,

where $\zeta(x)$ denotes the Riemann zeta function.

If the shape anisotropy favours the moments of the individual discs pointing perpendicular to the disc axis, the system exhibits a richer behaviour. The axial configuration, Ax, with the moments parallel to the wire axis now has an energy of

$E^{\mathrm{Ax}}=-J-4 \omega \zeta(3)$.

However, it might be favourable for the system to be in a state where the moments align perpendicular to the wire axis and form up or down spin domains of size $L$, where $L=1$ corresponds to anti-ferromagnetic and $L=\infty$ to ferromagnetic ordering. A straightforward calculation of the energies yields

$E^{L}=-J\left(1-\frac{2}{L}\right)-K+\omega \sigma(L)$,

where

$$
\begin{aligned}
\sigma(L)= & \frac{1}{L} \sum_{i=1}^{L}\left(\sum_{j=1, j \neq i}^{L} \frac{1}{|i-j|^{3}}\right. \\
& \left.-\sum_{j=1}^{L} \sum_{n=1}^{\infty}\left[\frac{(-1)^{n+1}}{|i-j-n L|^{3}}+\frac{(-1)^{n+1}}{|i-j+n L|^{3}}\right]\right) .
\end{aligned}
$$

Note that this takes values of $\sigma(1)=-\frac{3}{2} \zeta(3)$ and $\sigma(\infty)=2 \zeta(3)$ in the anti-ferromagnetic and ferromagnetic cases, respectively.

Comparing energies $E^{\mathrm{Ax}}$ and $E^{L}$, given in Eqs. (7) and (8), respectively, leads to the following boundaries between phases:

Between Ax and $L: \frac{K}{J}=\frac{2}{L}+\frac{\omega}{J}(2 \sigma(\infty)+\sigma(L))$,

Between $L_{1}$ and $L_{2}: \frac{\omega}{J}=2 \frac{1 / L_{1}-1 / L_{2}}{\sigma\left(L_{2}\right)-\sigma\left(L_{1}\right)}$, 
where $L_{1}$ and $L_{2}$ refer to the phase with domains of length $L_{1}$ and $L_{2}$, respectively. Thus, the ground state phase diagram is as shown in Fig. 2.

Interestingly, the analogue of the ferromagnetic, $\infty$, phase field in Fig. 2 does not exist for non-zero dipolar coupling $\omega$ in two dimensions [4]. However, in $1 \mathrm{~d}$, the dipolar sums converge more rapidly an it is rather straightforward to establish that this

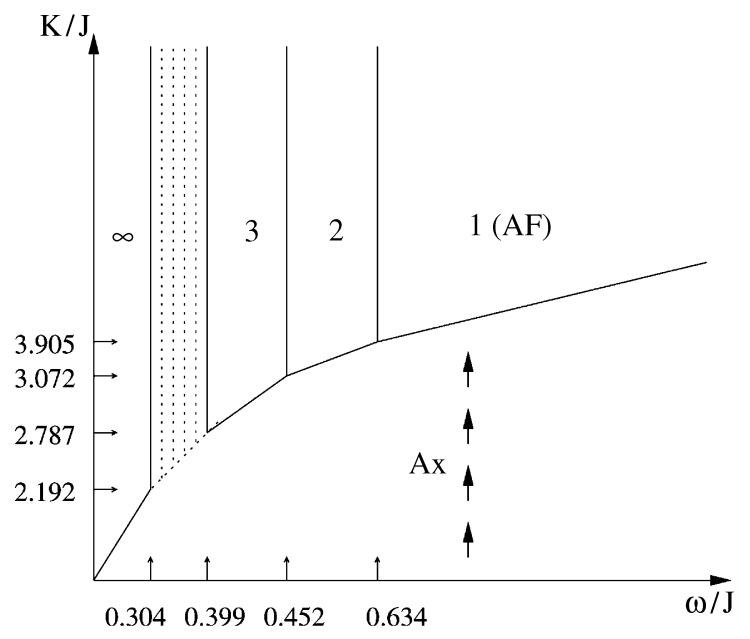

Fig. 2. Schematic ground state phase diagram for the full model Hamiltonian (1) with $J \neq 0$ under the constraints given in Eq. (5).

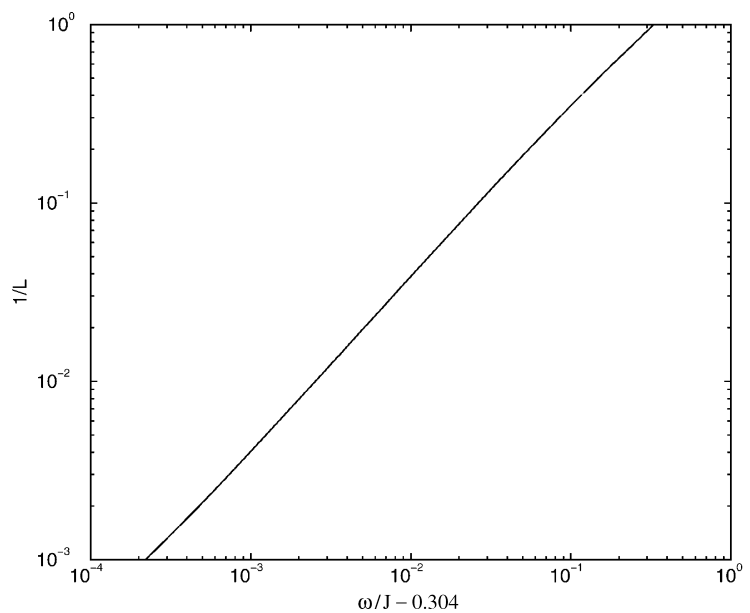

Fig. 3. Double logarithmic plot of inverse domain thickness $1 / L$ as a function of the strength of the dipolar interaction $\omega$. This suggests a behaviour $1 / L \approx A\left(\omega-\omega_{0}\right)^{v}$ where the numerically obtained value of the offset is $\omega_{0} / J \approx 0.304$ and $v \approx 1$. phase field has a finite width. As evidence in Fig. 3, we show a $\log -\log$ plot of $1 / L$, where $L$ is the ground state domain size, and the dipolar coupling constant $\omega$. Clearly, $L$ reaches infinity for finite $\omega$.

In this preliminary study of our model we shall focus on the AF to Ax transition. Evidently, the study of this problem is greatly simplified if the domains with $L \neq 1$ are eliminated as possible ground states. Clearly, this will be the case if the exchange interaction $J$, across the non-magnetic spacer, is taken to be zero. For the rest of the paper we shall consider this simplified case only. In this limit the phase diagram in Fig. 2 reduces to that shown in Fig. 4 where the boundary between the phases $A x$ and $L=1(\mathrm{AF})$ is given by

$K=\frac{5}{2} \omega \zeta(3)$.

Finally, to complete the discussion of the ground state, we have solved the magneto-statics problem for an individual disc, to determine the anisotropy constant $K$ as a function of aspect ratio $R / d$, and in Fig. 5 we display $K / \omega$ as a function of the separation $s$ between the discs. Clearly, with the help of this diagram the ratio $K / \omega$ can be adjusted, by changing the disc separation $s$, to be near the interesting critical value $(K / \omega)_{C}=$ $\frac{5}{2} \zeta(3)$.

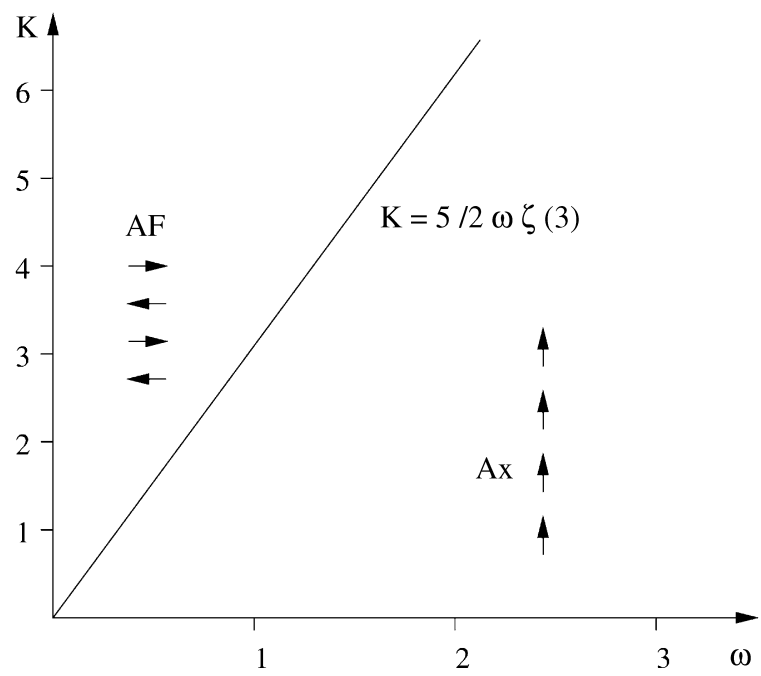

Fig. 4. Ground state phase diagram for $J=0$. 


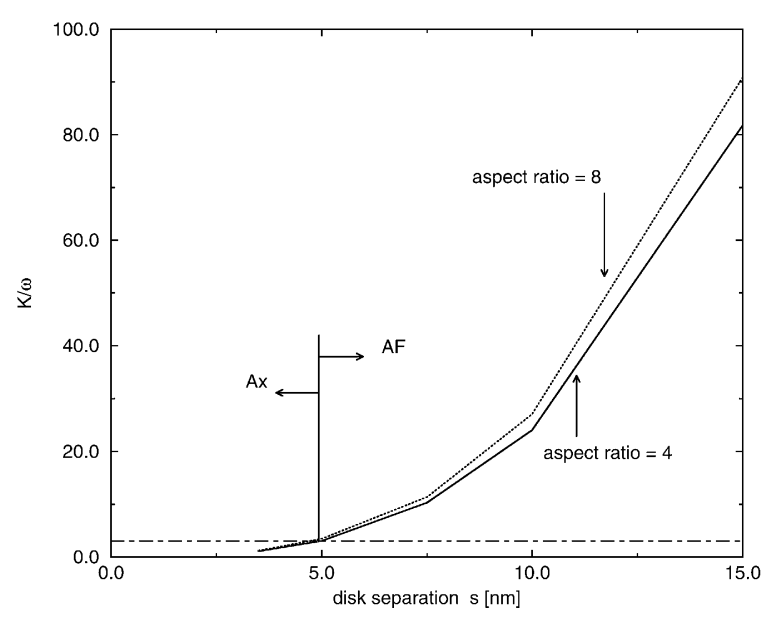

Fig. 5. Ratio of classical shape anisotropy $K$ and dipolar coup$\operatorname{ling} \omega$ as a function of the disc separation $s$ for cobalt discs. The discs radius $R$ is $40 \mathrm{~nm}$ and the aspect ratio $R / d 4$ and 8 , respectively.

\section{Mean field approximation}

Given the above variety of ground states it is of interest to investigate how they behave as the temperature is raised. To find a mean field description which preserves the important aspects of the problem, we approximate the full Hamiltonian by a sum of independent single-site terms

$H\left\{\boldsymbol{s}_{i}\right\}=\sum_{i} h_{i}(\{\boldsymbol{s}\}) \approx \sum_{i} h_{i}^{\mathrm{MF}}\left(\boldsymbol{s}_{i}\right)$,

where we replace the $s_{j}$ for $i \neq j$ by their expectation value $\boldsymbol{m}_{j}=\left\langle\boldsymbol{s}_{j}\right\rangle$. Thus, $h^{\mathrm{MF}}\left(\boldsymbol{s}_{i}\right)$ takes the form

$$
\begin{aligned}
h_{i}^{\mathrm{MF}}\left(\boldsymbol{s}_{i}\right)= & -J \boldsymbol{m}_{i-1} \cdot \boldsymbol{s}_{i}+K \sin ^{2} \theta_{i} \\
& +\omega \sum_{j \neq i} \frac{\boldsymbol{m}_{j} \cdot \boldsymbol{s}_{i}-3 m_{j}^{z} s_{i}^{z}}{|i-j|^{3}},
\end{aligned}
$$

where $\theta_{i}$ is the polar angle of $\boldsymbol{s}_{i}$. Note the quadratic term $K \sin ^{2} \theta_{i}$ arises from the fact that a variable $\boldsymbol{s}_{i}$ is never independent from itself. (i.e., $\left\langle\left(\boldsymbol{s}_{i}\right)^{2}\right\rangle$ $\neq\left\langle\boldsymbol{s}_{i}\right\rangle^{2}$ even if $\left\langle\boldsymbol{s}_{i} \cdot \boldsymbol{s}_{j}\right\rangle=\left\langle\boldsymbol{s}_{i}\right\rangle \cdot\left\langle\boldsymbol{s}_{j}\right\rangle$ for all $i \neq j$.) In the case of vanishing exchange interaction $(J=0)$, which we will assume for the following treatment, there will be no domain structure, so one may assume $\boldsymbol{m}_{j}=\boldsymbol{m}$ to be site independent in the ferromagnetic case. Then $h^{\mathrm{MF}}$ reduces to

$h_{i}^{\mathrm{MF}}\left(\boldsymbol{s}_{i}\right)=K \sin ^{2} \theta_{i}+2 \omega \zeta(3)\left[m^{x} s_{i}^{x}+m^{y} s_{i}^{y}-2 m^{z} s_{i}^{z}\right]$.

In the case of antiferromagnetic ordering there will be two sublattices: I and II with an opposite orientation of the magnetic moments, i.e. $\boldsymbol{m}_{\mathrm{I}}=-\boldsymbol{m}_{\mathrm{II}}$. This can be represented as $\boldsymbol{m}_{j}=(-1)^{j} \boldsymbol{m}$, thus leading to the following expression for $h^{\mathrm{MF}}$ :

$$
\begin{aligned}
h_{i}^{\mathrm{MF}}\left(\boldsymbol{s}_{i}\right)= & K \sin ^{2} \theta_{i}+(-1) \frac{i 3}{2} \omega \zeta(3) \\
& \times\left[m^{x} s_{i}^{x}+m^{y} s_{i}^{y}-2 m^{z} s_{i}^{z}\right] .
\end{aligned}
$$

With these assumptions the mean field partition function $Z^{\mathrm{MF}}$ takes the form $Z^{\mathrm{MF}}=\prod_{i} z_{i}$, where

$z_{i}=\int_{0}^{2 \pi} \mathrm{d} \phi \int_{0}^{\pi} \sin \theta \mathrm{d} \theta \exp \left(-\beta h_{i}^{\mathrm{MF}}(\theta, \phi)\right)$

and

$$
\begin{aligned}
h^{\mathrm{MF}}(\theta, \phi)= & K \sin ^{2} \theta \\
& +\left(2 \omega \zeta(3) m^{x}+H^{x}\right) \sin \theta \cos \phi \\
& +\left(2 \omega \zeta(3) m^{y}+H^{y}\right) \sin \theta \sin \phi \\
& +\left(-4 \omega \zeta(3) m^{z}+H^{z}\right) \cos \theta .
\end{aligned}
$$

Evidently, the theory is self-consistent if $m^{x}$ is given by

$m^{x}=\frac{1}{z_{0}} \int_{0}^{2 \pi} \mathrm{d} \phi \int_{0}^{\pi} \sin ^{2} \theta \cos \phi \mathrm{d} \theta \exp \left(-\beta h^{\mathrm{MF}}\right)$

and corresponding relations apply to $m^{y}$ and $m^{z}$.

To quantify the orientational distribution of the spins it is worthwhile to consider in addition to the averages $m^{x}, m^{y}$ and $m^{z}$ the expectation values

$$
\begin{aligned}
\left\langle\left(s^{x}\right)^{2}\right\rangle= & \frac{1}{z_{0}} \int_{0}^{2 \pi} \mathrm{d} \phi \\
& \times \int_{0}^{\pi} \sin ^{3} \theta \cos ^{2} \phi \mathrm{d} \theta \exp \left(-\beta h_{\mathrm{MF}}\right), \\
\left\langle\left(s^{y}\right)^{2}\right\rangle= & \frac{1}{z_{0}} \int_{0}^{2 \pi} \mathrm{d} \phi \\
& \times \int_{0}^{\pi} \sin ^{3} \theta \sin ^{2} \phi \mathrm{d} \theta \exp \left(-\beta h_{\mathrm{MF}}\right),
\end{aligned}
$$




$$
\begin{aligned}
\left\langle\left(s^{z}\right)^{2}\right\rangle= & \frac{1}{z_{0}} \int_{0}^{2 \pi} \mathrm{d} \phi \\
& \times \int_{0}^{\pi} \sin \theta \cos ^{2} \theta \mathrm{d} \theta \exp \left(-\beta h_{\mathrm{MF}}\right) .
\end{aligned}
$$

A very similar mean field theory was found to be useful in connection with layered magnetic structures by Jensen and Bennemann [5], Taylor and Györffy [6] and more recently by Hucht and Usadel [7].

In general evaluating these expressions for $m^{z},\left\langle\left(s^{z}\right)^{2}\right\rangle$ and $\left\langle\left(s^{x}\right)^{2}\right\rangle=\left\langle\left(s^{y}\right)^{2}\right\rangle$ at different ratios of the dipolar interaction $\omega$ and the anisotropy $K$ reveal the expected set of phase transitions: in Fig. 6 we describe an axial ferromagnetic state becoming a paramagnet and Fig. 7 corresponds to the demise of the (transverse) antiferromagnetic state, where $z_{i}$ is different on the two sublattices, namely $z_{\mathrm{I}}$ and $z_{\mathrm{II}}$.

However, a much richer structure is observed with parameters close to the ground state phase boundary in Fig. 4. As shown in Fig. 8 below the mean field transition temperature $T_{\mathrm{c}}^{\mathrm{MF}}$, the preferred spin fluctuations change from the $z$-direction to the $x-y$ plane.

Note that contrary to the above mean field results the exact solution of our model corresponds to no magnetic long-range order [8]. All the same,

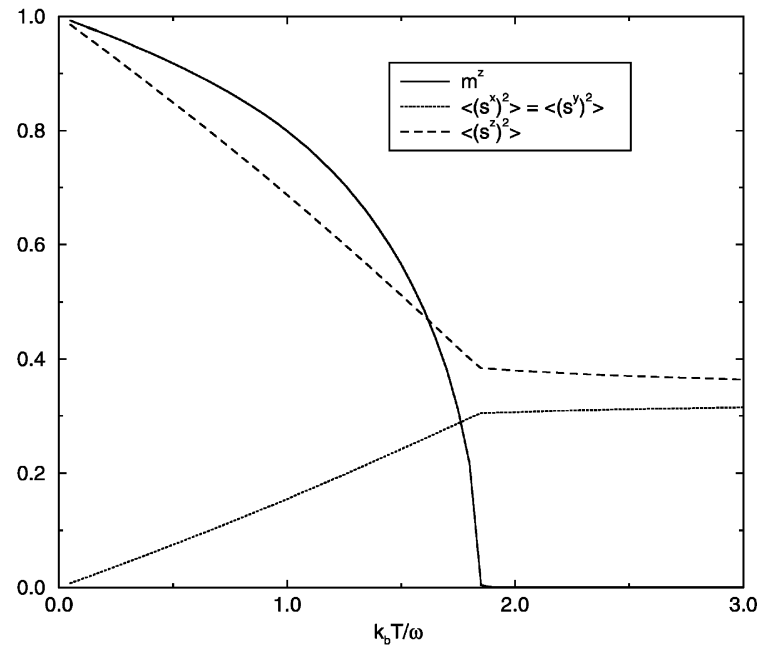

Fig. 6. Mean field results for $K=\omega$ for $J=0$. as the well-known example of the one-dimensional Ising model [9], recalled in the appendix, illustrates, the $T_{\mathrm{c}}^{\mathrm{MF}}$ does have a physical significance even in one-dimensional problems, where longrange order is forbidden by the Mermin-Wagner theorem [10]. It is that near $T_{\mathrm{c}}^{\mathrm{MF}}$ the thermal fluctuations of the magnetization slow down dramatically. Thus, while disregarding our results for the average magnetization $m^{x}, m^{y}$ and $m^{z}$ we can take

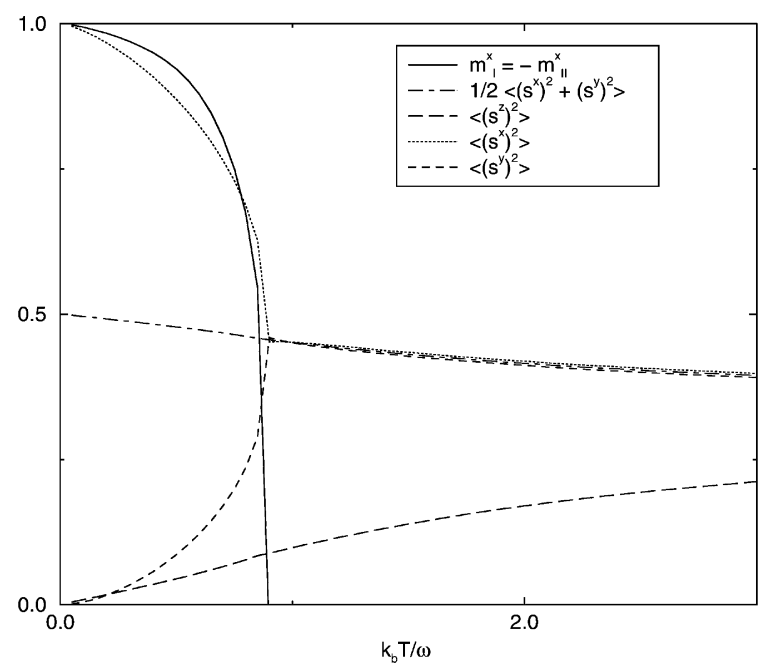

Fig. 7. Mean field results for $K=5 \omega$ for $J=0$.

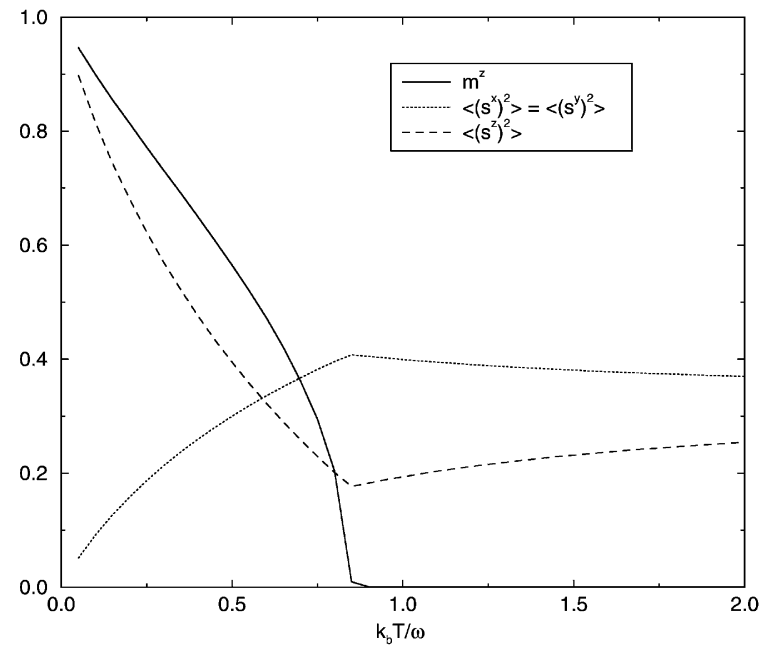

Fig. 8. Mean field results for $K=2 \omega$ for $J=0$. 
the predictions for $\left\langle\left(s^{x}\right)^{2}\right\rangle,\left\langle\left(s^{y}\right)^{2}\right\rangle$ and $\left\langle\left(s^{z}\right)^{2}\right\rangle$ seriously.

To lend support to the above argument, we have performed a number of Monte Carlo calculations for our model with $J=0$. We performed simulations of 1000 spins using Glauber dynamics with periodic boundary conditions. A typical simulation consists of 1000-10 000 Monte Carlo steps per spin. During the simulations we measured $\left\langle\left(s^{x}\right)^{2}\right\rangle$, $\left\langle\left(s^{y}\right)^{2}\right\rangle$, and $\left\langle\left(s^{z}\right)^{2}\right\rangle$. Figs. 9-11 show these ensemble

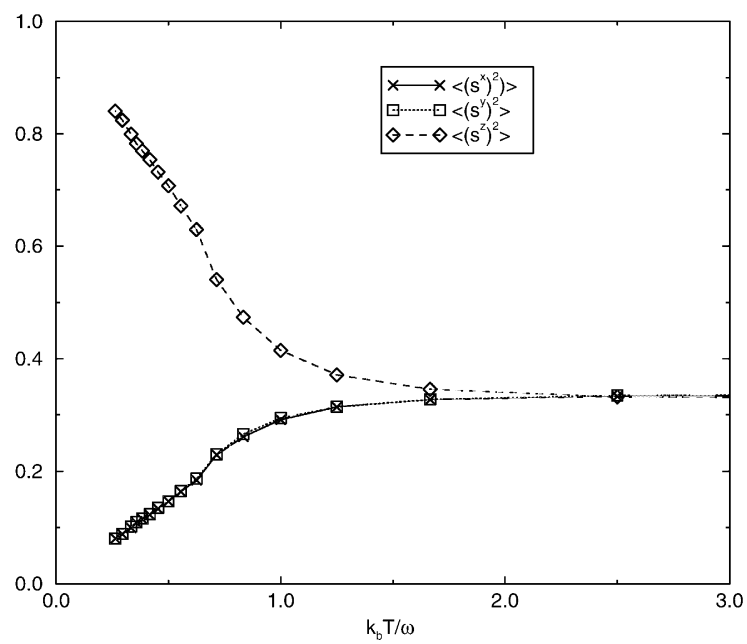

Fig. 9. Results from Monte Carlo simulation at $K=\omega$.

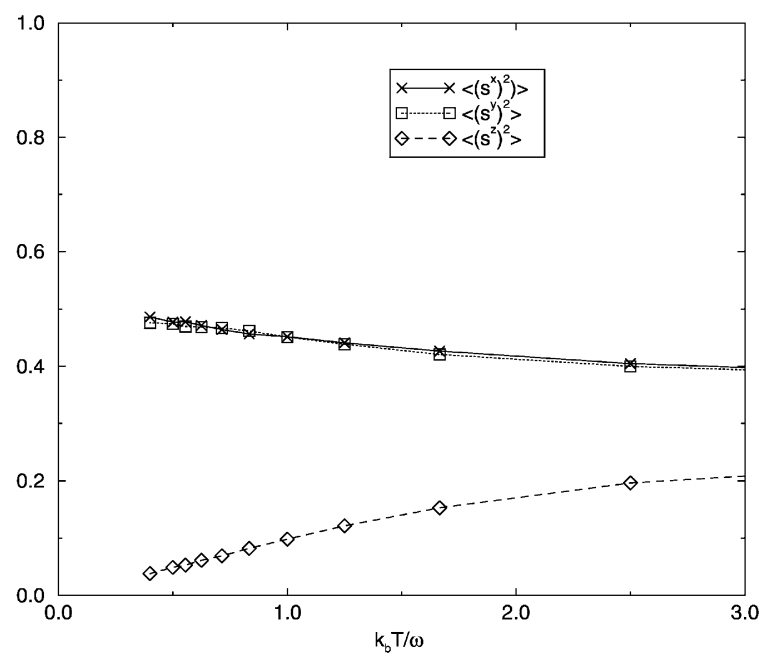

Fig. 10. Results from Monte Carlo simulation at $K=10 \omega$.

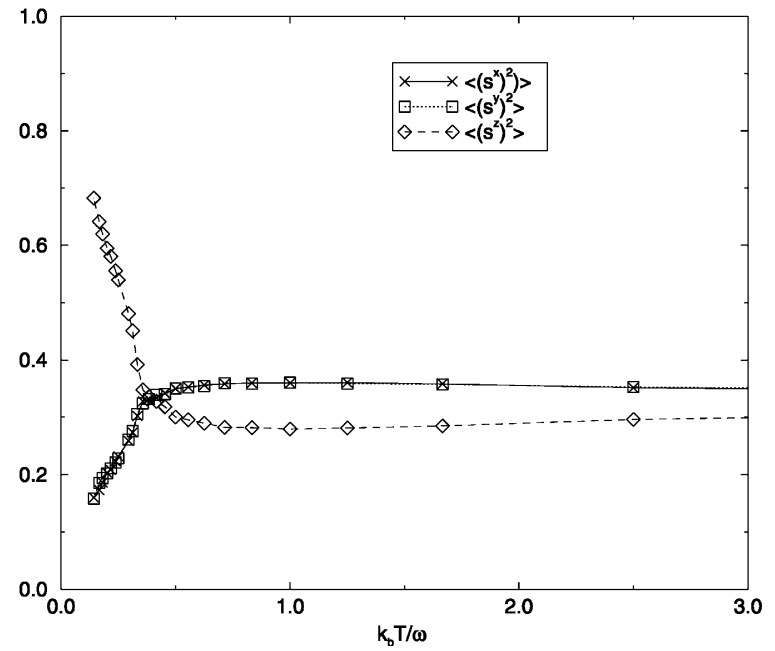

Fig. 11. Results from Monte Carlo simulation at $K=3 \omega$.

averages as a function of the temperature for $K=\omega, 3 \omega$, and $10 \omega$, respectively. Evidently, the simulations tell the same story as the mean field theory. Consequently, we do not expect the real system to exhibit spontaneous magnetization, but the spatial distribution of the magnetic fluctuation should show the general reorientational features we have found.

\section{Conclusion}

In summary, we have found a rich ground state structure for layered nanowires, that lies in the experimentally accessible region $(R \approx 30-40 \mathrm{~nm}$, $s, d \approx 5-10 \mathrm{~nm}$ for $\mathrm{Cu}$ /Co layers). Furthermore, we predict a temperature-dependent reorientation of the magnetic fluctuations that should manifest themself in the measurements of the axial and transverse susceptibilities of the wire.

\section{Appendix}

To underline the relevance of a mean field approach even in the case of an one-dimensional system we recall the results for the one-dimensional Ising model [9]

$H=-J \sum_{i} s_{i} s_{i+1}$ 

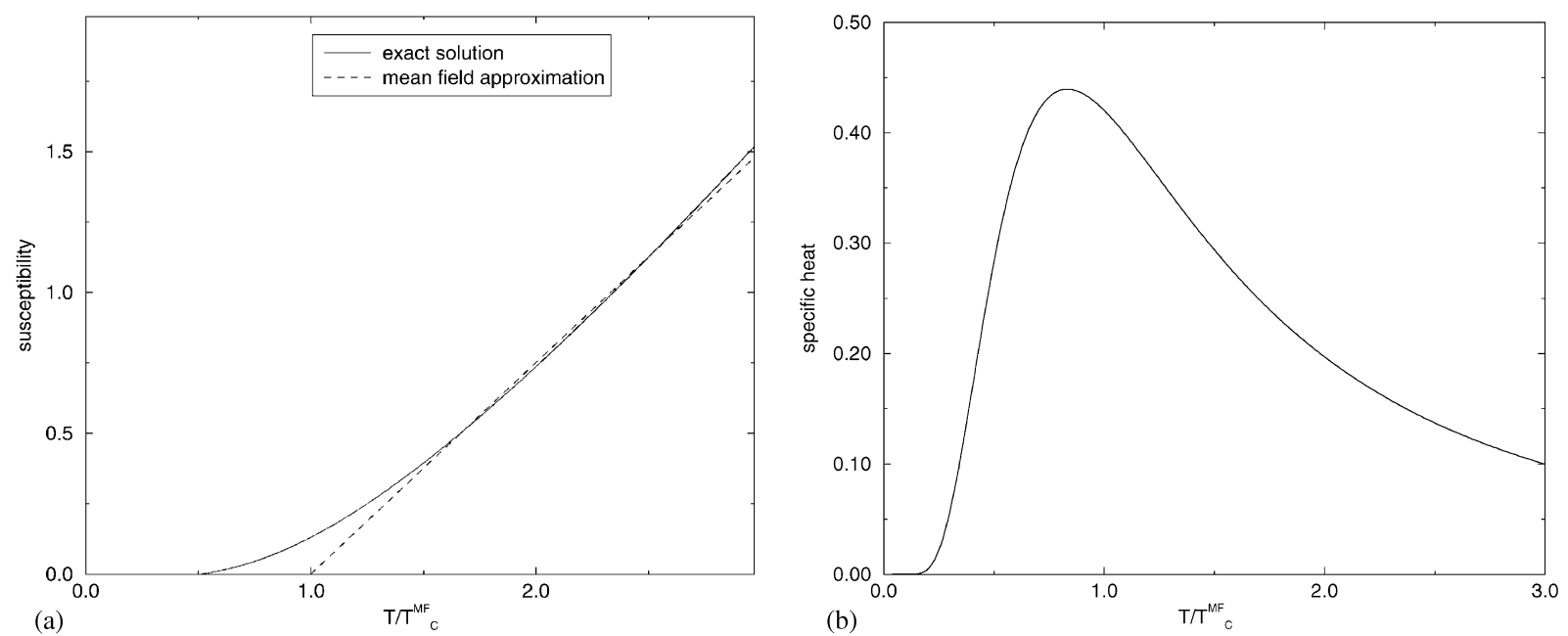

Fig. 12. Exact and mean field results for the one-dimensional Ising chain. (a) The exact (full line) and mean field (dotted line) susceptibilities. Note that even as the exact result exhibits no phase transition at finite temperature, a qualitative change in the behaviour, reminescent of the mean field phase transition, can be seen near $T_{\mathrm{C}}^{\mathrm{MF}}$. (b) The exact specific heat also shows a peak at a temperature near the mean field critical temperature.

The exact solutions for the susceptibility and specific heat are

$\chi=\beta \frac{1+\tanh \beta J}{1-\tanh \beta J}$

and

$C_{H}=(\beta J \operatorname{sech} \beta J)^{2}$.

In Fig. 12 we plot these and compare them to the corresponding mean field results. Evidently, the non-existence of a phase transition does not exclude a meaningful description of the change in the qualitative behaviour near the mean field critical temperature $T_{\mathrm{C}}^{\mathrm{MF}}$.

\section{References}

[1] R. Allenspach, J. Magn. Magn. Mater. 129 (1994) 160.

[2] W. Schwarzacher, D.S. Lashmore, IEEE Trans. Magn. 32 (1996) 3133.

[3] P. Bruno, Phys. Rev. B 52 (1995) 411.

[4] B. Kaplan, G.A. Gehring, J. Magn. Magn. Mater. 128 (1993) 111.

[5] P.J. Jensen, K.H. Bennemann, Phys. Rev. B 42 (1990) 849.

[6] M.B. Taylor, B.L. Györffy, J. Phys.: Condens. Matter 5 (1993) 4527.

[7] A. Hucht, K.D. Usadel, Phys. Rev. B 55 (1996) 12309.

[8] F.J. Dyson, Commun. Math. Phys. 12 (1969) 212.

[9] H.E. Stanley, Introduction to Phase Transitions and Critical Phenomena, Oxford University Press, Oxford, 1971, p. 87 and 121 .

[10] N.D. Mermin, H. Wagner, Phys. Rev. Lett. 17 (1966) 1133. 